\title{
Generalized hydrodynamics regime from the thermodynamic bootstrap program
}

\author{
Axel Cortés Cubero ${ }^{1}$, Miłosz Panfil ${ }^{2}$ \\ 1 Institute for Theoretical Physics, University of Amsterdam, Science Park 904, 1098 XH Amsterdam, \\ The Netherlands \\ 2 Faculty of Physics, University of Warsaw, Pasteura 5, 02-093 Warsaw, Poland \\ 1 a.cortescubero@uva.nl, 2 milosz.panfil@fuw.edu.pl
}

July 11, 2022

\begin{abstract}
Within the generalized hydrodynamics (GHD) formalism for quantum integrable models, it is possible to compute simple expressions for a number of correlation functions at the Eulerian scale. Specializing to integrable relativistic field theories, we show the same correlators can be computed as a sum over form factors, the GHD regime corresponding to the leading contribution with one particle-hole pair on a finite energy-density background. The thermodynamic bootstrap program (TBP) formalism was recently introduced as an axiomatic approach to computing such finite-energy-density form factors for integrable field theories. We derive a new axiom within the TBP formalism from which we easily recover the predicted GHD Eulerian correlators. We also compute higher form factor contributions, with more particle-hole pairs, within the TBP, allowing for the computation of correlation functions in the diffusive, and beyond, GHD regimes. The two particle-hole form factors agree with expressions recently conjectured within the GHD.
\end{abstract}

\section{Contents}

1 Introduction

2 Eulerian correlators from GHD and GHD with diffusion 
3 Thermodynamic bootstrap program

4 Single particle-hole form factors at low momentum

5 Two and more particle-hole pairs

6 Euler-scale correlators from particle-hole form factors

7 Conclusions

A Back-flow of the rapidities in the presence of a small particle-hole excitation

B Computation of the term $C^{2}$

\section{Introduction}

In this article, we explore the applications of the recently introduced Thermodynamic Bootstrap Program (TBP) [1 for correlation functions of integrable quantum field theories (IQFT) in the hydrodynamic regime. The thermodynamic bootstrap program is a set of axioms that strongly restrict form factors of physical operators between states of finite energy density, in IQFT's. One can then attempt to find exact expressions for these form factors, as self-consistent solutions of the set of axioms.

The TBP formalism is inspired by the standard integrable bootstrap program [2 4 , which is used to compute exact form factors involving a finite number of particles on top of the vacuum. The TBP generalizes this formalism to the case where there is a finite number of particle and hole excitations on top of a thermodynamic background (itself consisting of an infinite number of background particles), rather than the vacuum. The background state can be, in principle, any eigenstate of IQFT characterized by a smooth filling function, e.g. the thermal state [5] or GGE state 68. The thermodynamic form factors provides us with a fundamental ingredient for computation of the dynamic correlation functions in and out of the equilibrium.

Generalized Hydrodynamics (GHD) is an approach to study the dynamics of integrable models (in- 
cluding, but not limited to IQFT's) in spatially inhomogeneous setups. It is based on the idea that, given an inhomogeneous initial state, at late enough times, the spatial and temporal fluctuations are smooth enough, that they can be completely characterized by a set of hydrodynamical differential equations. It originated in [9, 10] as a way of solving the bi-partite quench protocol, in which two thermodynamically different systems are joined. Since then, it was developed into a coherent framework [11 17] capable of describing more general inhomogeneous setups in different integrable models $[9,11,18,26]$. Recently, the predictions of GHD were also confirmed experimentally [27].

The regime where GHD is applicable is that where only spatial fluctuations of very long wavelength are relevant. As shown in the original work [10] and also elucidated more recently [28], the crucial ingredient in formulating the GHD is then knowledge of the small momentum limit of form factors of conserved densities and currents over a thermodynamic background. This creates a point of overlap between TBP and GHD which we aim to explore in this work. Specifically, we show that the predictions of TBP agree with the assumed structure of these form factors which was put forward while formulating the GHD.

In particular, it was shown in [13,14,29, that the GHD formalism may be used to compute correlation functions of conserved charge and current densities in the hydrodynamical regime. Further, in 13 it was shown that for relativistic QFT's, Euler-scale correlators can be computed for general local operators. In the case of correlators of charge densities and currents, the same correlator can be computed as a sum over form factors over the thermodynamic background state. From the requirement that both expressions agree, one can derive from the GHD correlator, a simple prediction for low-momentum form factors of charge and current densities. We will show in this paper, that this prediction is easily recovered for IQFT's within the TBP formalism. Furthermore, we show that the general formula for any two local operators can be reproduced in the same way, by computing the one-particle-hole form factors.

Originally, the GHD was formulated at the Euler scales, where transport is generally ballistic. It was recently shown in [28,30] that diffusive behavior can be re-introduced by considering form factors with higher numbers of particle-hole pairs on top of the background, than those included in the hydrodynamical regime. This results in a Navier-Stokes GHD formulation. An important ingredient in this formulation is an assumption on the form of the leading 2-particle-hole pair form factor. The conjectured form that was put forward in 30 was inspired by the results of [31] for the thermodynamic limit of the density operator form factor in the non-relativistic integrable Lieb-Liniger model. Specifically, in formulating the NavierStokes GHD, it was assumed that the leading diverging part of the 2 particle-hole form factor is universal for any local operator. In this paper, we will see that this GHD conjecture is also easily recovered within the TBP formalism, in the relativistic QFT setting. The TBP also allows us to study the low-momentum limit form factors with even higher numbers of particle-hole pairs, going beyond the Navier-Stokes GHD 
conjecture. It is worth mentioning that guided by kinematical arguments, an equivalent formula for the Navier-Stokes GHD term was proposed in 32 .

The rest of the manuscript is organized in the following way. In section 2 we briefly review the predictions from GHD for correlation functions at the Euler scale, as well as the conjectures for GHD diffusion corrections. In section 3 we give a quick overview of the Thermodynamic Bootstrap program. In the following two sections we evaluate the leading form factors within the TBP formalism. First, in section 4 we evaluate the zero-momentum limit of the single particle-hole pair form factors for a generic local operator, which is the only necessary ingredient to compute Euler-scale correlators. Then, in section 5, we consider form factors with multi particle-hole excitations. The case of two particle-hole pairs is relevant for the inclusion of diffusion to the hydrodynamic picture. Finally, in section 6 , we consider a simple application of these results to the computation of Euler-scale correlation functions.

\section{Eulerian correlators from GHD and GHD with diffusion}

The GHD formalism allows one to study the long-time evolution of systems with spatially inhomogeneous initial conditions. Such initial states can be described as GGE-like states, but with spatially varying chemical potentials. Following the notation of [13], local observables in an initial state at $t=0$ are given by

$$
\langle\mathcal{O}\rangle_{\text {ini }}=\frac{\operatorname{Tr}\left(e^{-\int \mathrm{d} x \sum_{i} \beta^{i}(x) q^{i}(x)} \mathcal{O}\right)}{\operatorname{Tr}\left(e^{-\int \mathrm{d} x \sum_{i} \beta^{i}(x) q^{i}(x)}\right)},
$$

where $q^{i}(x)$ are the charge densities, corresponding to the conserved charges of the integrable model, $Q^{i}=\int \mathrm{d} x q^{i}(x)$ and $\beta^{i}(x)$ are local chemical potentials. One is then interested in computing space- and time-dependent correlation functions

$$
\left\langle\mathcal{O}\left(x_{1}, t_{1}\right) \ldots \mathcal{O}_{n}\left(x_{n}, t_{n}\right)\right\rangle_{\text {ini }}
$$

where the time evolution of operators is given by $\mathcal{O}(x, t)=e^{\mathrm{i} t H} \mathcal{O}(x, 0) e^{-\mathrm{i} t H}$.

The Eulerian scale, where hydrodynamical equations are applicable, of such correlation functions, is given by considering very late times, and large separations between operators (this scaling limit is more rigorously defined in [13]). At these scales, space and time dependence of the correlator can be captured by considering only the spatial and temporal dependence of chemical potentials in a local GGE. The time evolution of these chemical potentials is given by simple hydrodynamical differential equations. One 
central result of [13], is that at the Eulerian scale, correlation functions involving the charge density operators, $q_{i}(x)$, are given by

$$
\left\langle q^{i}(x, 0) \prod_{k} \mathcal{O}_{k}\left(x_{k}, t_{k}\right)\right\rangle_{\text {ini }}^{\text {Eulerian }}=-\frac{\delta}{\delta \beta^{i}(x)}\left\langle\prod_{k} \mathcal{O}_{k}\left(x_{k}, t_{k}\right)\right\rangle_{\text {ini }}^{\text {Eulerian }} .
$$

In other words, at the Eulerian scale, one can insert charge-density operators into correlation functions, simply by taking functional derivatives with respect to the corresponding chemical potential. A similar result also holds for the current density operators, $j^{i}(x, t)$, corresponding to the conserved charges.

Using knowledge of one-point functions for charge and current densities, and equation (3), the following two-point functions (among other results) at the Eulerian scale where proposed in [13

$$
\begin{aligned}
\left\langle q^{i}(x, t) q^{j}(0,0)\right\rangle_{\mathrm{ini}} & =\int \mathrm{d} \theta \delta\left(x-v^{\mathrm{eff}}(\theta) t\right) \rho_{p}(\theta)(1-\vartheta(\theta)) q_{\mathrm{dr}}^{i}(\theta) q_{\mathrm{dr}}^{j}(\theta), \\
\left\langle j^{i}(x, t) q^{j}(0,0)\right\rangle_{\mathrm{ini}} & =\int \mathrm{d} \theta \delta\left(x-v^{\mathrm{eff}}(\theta) t\right) \rho_{p}(\theta)(1-\vartheta(\theta)) v^{\mathrm{eff}}(\theta) q_{\mathrm{dr}}^{i}(\theta) q_{\mathrm{dr}}^{j}(\theta),
\end{aligned}
$$

where the functions $\rho(\theta)$ and $\vartheta(\theta)$ describe the distribution of the background particles in the GGE ensemble, $q_{\mathrm{dr}}^{i}(\theta)$ is the dressed eigenvalue of the charge $Q^{i}$ for a particle of rapidity $\theta$ on top of the background, and $v^{\text {eff }}(\theta)$ is the velocity of a particle of rapidity $\theta$, dressed by the background. All these quantities will be defined in more detail in the following section. The results (4), as written, are valid for a homogeneous initial state, $\beta^{i}(x)=\beta^{i}$. This will suffice for the comparison with the TBP. The GHD provides a way to lift these expressions to an inhomogeneous setup. We refer again to 13 for details.

For integrable QFT's, within the GHD formalism, it was shown in 13 that Euler-scale correlation functions can be computed for arbitrary local operators. The procedure is to start from the known expression for the one-point correlation function of an operator in a GGE, $\langle\mathcal{O}(x, t)\rangle_{\mathrm{GGE}}$, which can be computed through the LeClair-Mussardo formula [33, 34]. Then the charge density-generic operator Eulerian two-point function can be computed through Eq. (3). One is then able to extract from this correlator what is the contribution corresponding to each, the charge density, and the generic operator. Extracting and isolating the contribution from the generic operator, one can then write the general two point function (for a spatially homogeneous GGE state)

$$
\lim _{t \sim x \rightarrow \infty}\left\langle\mathcal{O}_{1}(x, t) \mathcal{O}_{2}(0,0)\right\rangle_{\vartheta}-\left\langle\mathcal{O}_{1}\right\rangle_{\vartheta}\left\langle\mathcal{O}_{2}\right\rangle_{\vartheta}=\int d \theta \delta\left(x-v^{\mathrm{eff}}(\theta) t\right) \rho_{p}(\theta)(1-\vartheta(\theta)) V^{\mathcal{O}_{1}}(\theta) V^{\mathcal{O}_{2}}(\theta)
$$

where $V^{\mathcal{O}}(\theta)$ are operator-specific functions derived from the Leclair-Mussardo formula as

$$
V^{\mathcal{O}}(\theta)=\sum_{k=0}^{\infty} \frac{1}{k !} \int \prod_{j=1}^{k}\left(\frac{d \theta_{j}}{2 \pi} \vartheta\left(\theta_{j}\right)\right)\left(2 \pi \rho_{s}(\theta)\right)^{-1} f_{c}^{\mathcal{O}}\left(\theta_{1}, \ldots, \theta_{k}, \theta\right)
$$


and $f_{c}^{\mathcal{O}}(\{\theta\})$ are the connected form factors of the operator, $\mathcal{O}$, which we will define more precisely in Section 4. In writing (5), we have used the effective velocity defined as $v^{\text {eff }}(\theta)=\left(E^{\prime}\right)^{\mathrm{dr}}(\theta) /\left(p^{\prime}\right)^{\mathrm{dr}}(\theta)$, where the "dressing" procedure for the energy and momentum are explained in more detail in the next section. The integral in (5) localizes to countable number of contributions given by $\theta_{*}(\xi)$ which are solutions of the equation $v^{\text {eff }}(\theta)=\xi$, where $\xi \equiv x / t$,

$$
\lim _{t \sim x \rightarrow \infty}\left\langle\mathcal{O}_{1}(x, t) \mathcal{O}_{2}(0,0)\right\rangle_{\vartheta}-\left\langle\mathcal{O}_{1}\right\rangle_{\vartheta}\left\langle\mathcal{O}_{2}\right\rangle_{\vartheta}=t^{-1} \sum_{\theta \in \theta_{*}(\xi)} \frac{\rho_{p}(\theta)(1-\vartheta(\theta))}{\left|\left(v^{\mathrm{eff}}\right)^{\prime}(\theta)\right|} V^{\mathcal{O}_{1}}(\theta) V^{\mathcal{O}_{2}}(\theta)
$$

We will show explicitly in Section 6 how expression (7) can be fully recovered within the TBP formalism, by including only form factors with one particle-hole pair with the same rapidity on top of the thermodynamic background.

It was recently shown in 28, 30] that diffusive behavior can be reintroduced into the GHD formalism by introducing into the hydrodynamic equations a term proportional to the diffusion matrix, defined as

$$
(\mathcal{D} C)_{i j}=\int d t\left[\int \mathrm{d} x\left\langle j^{i}(x, t) j^{j}(0,0)\right\rangle_{\vartheta}^{\text {connected }}-\left(\lim _{t^{\prime} \rightarrow \infty} \int \mathrm{d} x\left\langle j^{i}\left(x, t^{\prime}\right) j^{j}(0,0)\right\rangle_{\vartheta}^{\text {connected }}\right)\right] .
$$

If one were to compute such diffusion matrix in terms of a form factor expansion, the first non-trivial contribution would come from the low-momentum limit of the two particle-hole pair expansion (whereas the Eulerian scale involves only one particle-hole pair). It has been argued that the form of this diffusion matrix at this leading order should be

$$
\begin{aligned}
(\mathcal{D C})_{i j}= & \int \frac{\mathrm{d} \theta \mathrm{d} \alpha}{2} \rho_{p}(\theta) f(\theta) \rho_{p}(\alpha) f(\alpha)\left|v_{\mathrm{dr}}(\theta)-v_{\mathrm{dr}}(\alpha)\right| \\
& \times\left(\frac{q_{\mathrm{dr}}^{i}(\theta) T^{\mathrm{dr}}(\theta, \alpha)}{\rho_{s}(\theta)}-\frac{q_{\mathrm{dr}}^{i}(\alpha) T^{\mathrm{dr}}(\alpha, \theta)}{\rho_{s}(\alpha)}\right)\left(\frac{q_{\mathrm{dr}}^{j}(\theta) T^{\mathrm{dr}}(\theta, \alpha)}{\rho_{s}(\theta)}-\frac{q_{\mathrm{dr}}^{j}(\alpha) T^{\mathrm{dr}}(\alpha, \theta)}{\rho_{s}(\alpha)}\right),
\end{aligned}
$$

where $\rho_{s}(\theta)$ is the density of states describing the thermodynamic background, and $T^{\mathrm{dr}}(\theta, \alpha)$ is related to the dressed scattering phase (we will define more clearly both functions in the following sections), and $f(\theta)$ is a statistical factor, depending on whether particles are bosonic or fermionic. From this form of the diffusion matrix, which should be given by the two particle-hole pair form factor of the current operator, a general expression for this form factor was conjectured in Ref. [30]. We will show how this conjectured form factor can be derived explicitly for IQFT's in the TBP formalism, reproducing exactly the expected diffusion matrix. 


\section{Thermodynamic bootstrap program}

In this section we provide a short summary of the Thermodynamic Bootstrap Program. For a detailed presentation, we refer to [1]. The aim of the TBP is to provide a formalism for computing form factors in IQFTs at finite energy density. The existence of an infinite number of local conserved charges in IQFT's results in the absence of creation and annihilation processes and factorization of any $n$-body scattering process into a product of 2-body scattering processes [4]. The IQFT's are characterized by the elastic 2-body scattering matrix $S\left(\theta_{1}-\theta_{2}\right)$, where the rapidity is defined as the parametrization of the on-shell energy and momentum of particles, $E=m \cosh (\theta), p=m \sinh (\theta)$. For the remainder of this paper, we will consider IQFT's with only one species of particles of mass $m$, for simplicity of presentation, but our results could be generalized for theories with a richer spectrum.

The states at finite energy density, such as the thermal states, are described by specifying the filling function $\vartheta(\theta)$, describing the probability of finding a particle of rapidity $\theta$ on the state. Due to the interactive nature of the theory, the physical density of the particles, $\rho_{p}(\theta)$, depends on the presence of other particles and follows from the integral equation

$$
\rho_{s}(\theta)=m \cosh (\theta)+\int \mathrm{d} \theta^{\prime} T\left(\theta, \theta^{\prime}\right) \rho_{p}\left(\theta^{\prime}\right), \quad \rho_{p}(\theta)=\vartheta(\theta) \rho_{s}(\theta),
$$

where $\rho_{s}(\theta)$ is the total density of particles and $T\left(\theta, \theta^{\prime}\right)$ is the scattering kernel related to the $S$-matrix 1 ,

$$
T\left(\theta, \theta^{\prime}\right)=\frac{1}{2 \pi} \frac{\partial}{\partial \theta} \delta\left(\theta-\theta^{\prime}\right), \quad \delta(\theta)=-i \log (-S(\theta)) .
$$

The energy density (for system size $L$ ) of a state is

$$
\frac{E}{L}=\int \mathrm{d} \theta \rho_{p}(\theta) \cosh (\theta)
$$

and expectation values of other conserved charges are

$$
\left\langle Q^{i}\right\rangle_{\vartheta}=\frac{\left\langle\vartheta\left|Q^{i}\right| \vartheta\right\rangle}{\langle\vartheta \mid \vartheta\rangle}=L \int \mathrm{d} \theta \rho_{p}(\theta) q^{i}(\theta)
$$

where $q^{i}(\theta)$ is the charge eigenvalue on a one-particle state, $Q^{i}|\theta\rangle=q^{i}(\theta)|\theta\rangle$.

We define a finite-energy density state, $|\vartheta\rangle$, as an eigenstate with an extensive number of particles, whose rapidities are distributed according to the filling fraction $\vartheta(\theta)$. We then consider excitations on top of this thermodynamic background, by adding or removing a finite number of particles, characterized by

\footnotetext{
${ }^{1}$ To agree with the GHD notation we use here a slightly different notation than in 1 . Namely, the integral equations are written in terms of the kernel $T\left(\theta, \theta^{\prime}\right)$, instead the standard IQFT notation uses $\varphi\left(\theta, \theta^{\prime}\right)=2 \pi T\left(\theta, \theta^{\prime}\right)$.
} 
a set of rapidities $\left\{\theta_{j}\right\}_{j=1}^{n}$. We denote such excited states by $\left|\vartheta ; \theta_{1}, \ldots, \theta_{n}\right\rangle$. Again, due to the interactive nature of the theory, the excited state not only has extra particles, but also the distribution of the background particles $\vartheta(\theta)$ is slightly shifted. This shift is described by the back-flow function

$$
\begin{aligned}
F(\theta \mid\{\theta\}) & =\sum_{j=1}^{n} F\left(\theta \mid \theta_{j}\right), \\
F\left(\theta \mid \theta_{j}\right) & =\frac{1}{2 \pi} \delta\left(\theta-\theta_{j}\right)+\int \mathrm{d} \theta^{\prime} T\left(\theta, \theta^{\prime}\right) \vartheta\left(\theta^{\prime}\right) F\left(\theta^{\prime} \mid \theta_{j}\right),
\end{aligned}
$$

which dictates the amount by which the rapidity of a background particle, originally located at $\theta$, is shifted by the introduction of a set of excitations with rapidities $\{\theta\}$. In the limit of the low momentum particle-hole excitation the back-flow is effectively captured by dressed differential scattering phase

$$
T^{\mathrm{dr}}\left(\theta, \theta_{1}\right)=T\left(\theta, \theta_{1}\right)+\int \mathrm{d} \theta^{\prime} \vartheta\left(\theta^{\prime}\right) T\left(\theta^{\prime}, \theta\right) T^{\mathrm{dr}}\left(\theta, \theta_{1}\right)
$$

The effect of the shift on the expectation values of conserved charges can be encapsulated by the dresing procedure. The expectation values of a conserved charge $Q^{i}$ on a state $\left|\vartheta ; \theta_{1}, \ldots, \theta_{n}\right\rangle$ is

$$
\frac{\left\langle\vartheta ; \theta_{1}, \ldots, \theta_{n}\left|Q^{i}\right| \vartheta ; \theta_{1}, \ldots, \theta_{n}\right\rangle}{\left\langle\vartheta ; \theta_{1}, \ldots, \theta_{n} \mid \vartheta ; \theta_{1}, \ldots, \theta_{n}\right\rangle}=\left\langle Q^{i}\right\rangle_{\vartheta}+q_{\mathrm{eff}}^{i}\left(\theta_{1}\right)+\cdots+q_{\mathrm{eff}}^{i}\left(\theta_{n}\right)
$$

where we define the effective charge as the bare charge of a particle, $q(\theta)$ plus the shift in the value of the background's charge, induced by the presence of the new particle, or

$$
q_{\mathrm{eff}}(\theta)=q(\theta)-\int d \theta^{\prime} \vartheta\left(\theta^{\prime}\right) q^{\prime}\left(\theta^{\prime}\right) F\left(\theta^{\prime} \mid \theta\right)
$$

We will later use proper names, $k(\theta)$ and $\omega(\theta)$ to refer to the effective momentum and energy of an excitation. The derivative of the effective charge can be shown to satisfy

$$
\left(q_{\mathrm{eff}}^{i}\right)^{\prime}(\theta)=\left(q^{i}\right)^{\prime}(\theta)+\int \mathrm{d} \theta^{\prime} T\left(\theta, \theta^{\prime}\right) \vartheta\left(\theta^{\prime}\right)\left(q_{\mathrm{eff}}^{i}\right)^{\prime}\left(\theta^{\prime}\right)
$$

From this integral equation (19), it is useful to define the "dressing" procedure, for a general function $g(\theta)$, producing a dressed function as

$$
g_{\mathrm{dr}}(\theta)=g(\theta)+\int \mathrm{d} \theta^{\prime} T\left(\theta, \theta^{\prime}\right) \vartheta\left(\theta^{\prime}\right) g_{\mathrm{dr}}\left(\theta^{\prime}\right),
$$

or written in operatorial form

$$
g(\theta)=\left((1-T \vartheta) g_{\mathrm{dr}}\right)(\theta)
$$

Its formal solution is

$$
g_{\mathrm{dr}}(\theta)=\left((1-T \vartheta)^{-1} g\right)(\theta)
$$


with the dressing operator $(1-T \vartheta)^{-1}$. For example, the dressed momentum and energy, follow from the dressing of the single particle expectation values $m \sinh \theta$ and $m \cosh \theta$ respectively. Another example of the dressing procedure in action is the integral equation (16).

Form factors of local operators within the standard integrable bootstrap program are defined as the functions,

$$
f^{\mathcal{O}}\left(\theta_{1}, \ldots, \theta_{n}\right) \equiv\left\langle 0|\mathcal{O}| \theta_{1}, \ldots, \theta_{n}\right\rangle
$$

The bootstrap program consists in formulating a set of axioms that these functions satisfy, and which strongly constrain the form factors. The end goal is to have enough constraints such that the functions $f^{\mathcal{O}}\left(\theta_{1}, \ldots, \theta_{n}\right)$ can be recovered analytically as the minimal function that consistently satisfies all the axioms [2, 4].

The main goal of the TBP is to generalize the concept of form factor axioms to the case where particle and hole excitations are on top of a (generalized) thermodynamic background, rather than on top of the vacuum, as in the standard bootstrap program. We define now a form factor of a local operator $\mathcal{O}(x)$ as the function

$$
f_{\vartheta}^{\mathcal{O}}\left(\theta_{1}, \ldots, \theta_{n}\right)=\frac{\left\langle\vartheta|\mathcal{O}(0)| \vartheta ; \theta_{1}, \ldots, \theta_{n}\right\rangle}{\langle\vartheta \mid \vartheta\rangle} .
$$

These form factors need to be properly defined by first considering a finite volume expression with a finite number of particles, then taking the thermodynamic limit. We will confront this issue in more detail in the next section. In relativistic field theories, introducing a hole excitation (or removing a particle excitation) of rapidity $\theta$ is equivalent to introducing a particle with rapidity $\theta+\pi \mathrm{i}$, such that the expression (24) can describe both, particle and hole excitations. The shift in rapidity $\theta+\pi \mathrm{i}$ can also be interpreted in terms of crossing symmetry, as adding a particle of rapidity $\theta$ in the bra, instead of the ket state.

These form factors enter the expression for the two-point correlation function [1]

$$
\begin{aligned}
\frac{\langle\vartheta|\mathcal{O}(x, t) \mathcal{O}(0,0)| \vartheta\rangle}{\langle\vartheta \mid \vartheta\rangle}= & \sum_{n=0} \sum_{\sigma_{i}= \pm 1}\left(\prod_{k=1}^{n} f_{-\infty}^{\infty} \frac{\mathrm{d} \theta}{2 \pi} \vartheta_{\sigma_{k}}\left(\theta_{k}\right)\right) f_{\vartheta}^{\mathcal{O}}\left(\theta_{1}, \ldots, \theta_{n}\right)_{\sigma_{1}, \ldots, \sigma_{n}}\left(f_{\vartheta}^{\mathcal{O}^{\prime}}\left(\theta_{1}, \ldots, \theta_{n}\right)_{\sigma_{1}, \ldots, \sigma_{n}}\right)^{*} \\
& \times \exp \left(\mathrm{i} x \sum_{k=1}^{n} \sigma_{k} k\left(\theta_{k}\right)-\mathrm{i} t \sum_{k=1}^{n} \sigma_{k} \omega\left(\theta_{k}\right)\right)
\end{aligned}
$$

where we define the filling fractions,

$$
\vartheta_{-1}\left(\theta_{k}\right)=\vartheta\left(\theta_{k}\right), \quad \vartheta_{+1}\left(\theta_{k}\right)=\frac{\rho_{h}(\theta)}{\rho_{p}(\theta)} \vartheta\left(\theta_{k}\right) .
$$


and

$$
f_{\vartheta}^{\mathcal{O}}\left(\theta_{1}, \ldots, \theta_{n}\right)_{\sigma_{1}, \ldots, \sigma_{n}}=f_{\vartheta}^{\mathcal{O}}\left(\theta_{1}+i \pi \delta_{\sigma_{1},-1}, \ldots, \theta_{n}+i \pi \delta_{\sigma_{n},-1}\right)
$$

Functions $k(\theta)$ and $\omega(\theta)$ are the effective energy and momentum, respectively, of a particle of rapidity $\theta$. The two-point function is written as an expansion in terms of the dressed form factors, with particle and hole excitations on top of the background, $\vartheta$. We use $\sigma$ to distinguish particles $(\sigma=1)$ from holes $(\sigma=-1)$. Moreover, behind the $f$ symbol there is a prescription for the integration which should be performed along a line shifted by $i \epsilon$ above the real axis and with the residues from the double annihilation poles subtracted.

The formula 25] corrects some issues with the previously conjectured Leclair-Mussardo formula [35]. In particular, it involves the new form factors that have been dressed by the thermodynamic background, instead of the vacuum form factors used in [35]. The formula (25) incorporates also a precise prescription to deal with the singularities arising from the form factors, whereas in the proposal of 35 no regularization procedure was proposed. An alternate regularization procedure for the Leclair Mussardo formula was recently proposed in [36]. The formula proposed in [36] can in practice only be computed as a low temperature expansion which sets it far from the GHD regime. Finally, we note that within the TBP the axioms are formulated in the microcanonical approach using the concept of a representatitve state. An approach based on formulating axioms for the genuine Gibbs ensemble, put forward in 37 for Ising field theory, turned out to be difficult to generalize to interacting IQFT's.

In [1] we postulated that form factors (24) obey a set of axioms. We will not list all of these here, but only show some of the most relevant ones to our discussion, which are

- Scattering axiom

$$
f_{\vartheta}^{\mathcal{O}}\left(\theta_{1}, \ldots, \theta_{i}, \theta_{i+1}, \ldots, \theta_{n}\right)=S\left(\theta_{i}-\theta_{i=1}\right) f_{\vartheta}^{\mathcal{O}}\left(\theta_{1}, \ldots, \theta_{i+1}, \theta_{i}, \ldots, \theta_{n}\right)
$$

- Periodicity axiom

$$
f_{\vartheta}^{\mathcal{O}}\left(\theta_{1}, \ldots, \theta_{n}\right)=R_{\vartheta}\left(\theta_{n} \mid \theta_{1}, \ldots, \theta_{n}\right) f_{\vartheta}^{\mathcal{O}}\left(\theta_{n}+2 \pi i, \theta_{1}, \ldots, \theta_{n-1}\right)
$$

- Annihilation pole axiom

$$
\begin{aligned}
& -i \operatorname{Res}_{\theta_{1}=\theta_{2}} f_{\vartheta}^{\mathcal{O}}\left(\theta_{1}, \theta_{2}, \ldots, \theta_{n}\right)= \\
& \quad\left[1-R_{\vartheta}\left(\theta_{2} \mid \theta_{3}, \ldots, \theta_{n}\right) S\left(\theta_{2}-\theta_{3}\right) \times \cdots \times S\left(\theta_{2}-\theta_{n}\right)\right] f_{\vartheta}^{\mathcal{O}}\left(\theta_{3}, \ldots, \theta_{n}\right)
\end{aligned}
$$


- Spatial and temporal translation

$$
f_{\vartheta}^{\mathcal{O}}(x, t)\left(\theta_{1}, \ldots, \theta_{n}\right) \equiv \frac{\left\langle\vartheta|\mathcal{O}(x, t)| \vartheta ; \theta_{1}, \ldots, \theta_{2}\right\rangle}{\vartheta \mid \vartheta}=e^{\mathrm{i} x m \sum_{i=1}^{n} k\left(\theta_{i}\right)-\mathrm{i} t m \sum_{i=1}^{n} \omega\left(\theta_{i}\right)} f_{\vartheta}^{\mathcal{O}}\left(\theta_{1}, \ldots, \vartheta\right),
$$

Here, $R_{\vartheta}\left(\theta \mid \theta_{1}, \ldots, \theta_{n}\right)$ is related to the back-flow function through

$$
\begin{aligned}
R_{\vartheta}\left(\theta \mid \theta_{1}, \ldots, \theta_{n}\right) & =\prod_{j=1}^{n} R_{\vartheta}\left(\theta \mid \theta_{j}\right) \\
R_{\vartheta}\left(\theta \mid \theta_{j}\right) & =\exp \left(2 \pi i F\left(\theta \mid \theta_{j}\right)-i \delta\left(\theta-\theta_{j}\right)\right) .
\end{aligned}
$$

The presented axioms generalize the vacuum axioms [2,3] and reduce to them in the $\vartheta(\theta) \rightarrow 0$ limit. In this limit, $R_{0}\left(\theta \mid \theta_{j}\right)=1$.

We will see later, in section 6 , that when computing correlation functions of local operators at the Euler scale, we only need to consider the leading contribution to the expansion (25). This contribution is given by considering only form factors with one particle-hole pair excitation on top of it, in the zeromomentum limit (in the limit where the rapidity of the particle and the hole coincide, such that the momentum and energy of the pair vanishes). That is, the only ingredient we need to compute Euler-scale correlators are form factors of the form $\lim _{\kappa \rightarrow 0} f_{\vartheta}^{\mathcal{O}}(\theta+\pi \mathrm{i}, \theta+\kappa)$. In the next section we evaluate and find an analytic expression for such form factors.

The annihilation pole axiom will be of particular importance to our discussion in the section 5 . This axiom controls the form factor in the region where a particle-hole pair has rapidities very close to each other, in the presence of other excitations. This region is important for obtaining diffusive corrections to the GHD dynamics.

Finally, we note that the form factors in the GHD convention 30 differ from our convention by appearance of extra factors $\rho_{s}\left(\theta_{j}\right)$. For a form factor with $n$ particle-hole excitations

$$
f_{\vartheta}^{\mathcal{O}}\left(\theta_{1}^{+}, \ldots, \theta_{n}^{+}, \theta_{1}^{-}+i \pi, \ldots, \theta_{n}^{-}+i \pi\right)=\frac{\left\langle\vartheta|\mathcal{O}| \vartheta ;\left\{\theta_{j}^{+}, \theta_{j}^{-}\right\}\right\rangle_{\mathrm{GHD}}}{\sqrt{\prod_{j=1}^{n} 2 \pi \rho_{s}\left(\theta_{j}^{+}\right) 2 \pi \rho_{s}\left(\theta_{j}^{-}\right)}} .
$$

\section{Single particle-hole form factors at low momentum}

In this section, we are interested in the evaluation of the form factor

$$
\lim _{\kappa \rightarrow 0} f_{\vartheta}^{\mathcal{O}}(\theta+\pi \mathrm{i}, \theta+\kappa)
$$


In particular, we will obtain an expression for this form factor in terms of the standard, zero-background form factors.

Our first step is to regularize the form factor by studying its leading contributions at a large but finite volume, $L$. At finite volume, the particle rapidities are quantized, given by the solutions, $\{\tilde{\theta}\}$ of the Bethe equations [4]

$$
Q_{k}\left(\theta_{1}, \ldots, \theta_{n}\right) \equiv L m \sinh \theta_{k}+\sum_{l \neq k} \delta\left(\theta_{k}-\theta_{l}\right)=2 \pi I_{k},
$$

for each value of $k \in[1, n]$. The numbers $\{I\}$ are a set of integers (or half integers, depending on the particle statistics), for each particle. The Bethe equations then serve as a mapping from a set of quantum numbers $\{I\}$ into a set of of rapidities $\{\theta\}$.

At finite volume, it is then convenient to express form factors in the basis of the set of integers $\{I\}$. Following the results of $38[40]$, the finite-volume form factors can be expressed in terms of the infinite-volume form factors, as

$$
\left\langle I_{1}^{\prime}, \ldots, I_{m}^{\prime}|O(0)| I_{1}, \ldots, I_{n}\right\rangle=\frac{f^{\mathcal{O}}\left(\theta_{1}^{\prime}+\pi \mathrm{i}, \ldots, \theta_{m}^{\prime}+\pi \mathrm{i}, \theta_{1}, \ldots, \theta_{n}\right)}{\sqrt{\rho_{m}\left(\theta_{1}^{\prime}, \ldots, \theta_{m}^{\prime}\right) \rho_{n}\left(\theta_{1}, \ldots, \theta_{n}\right)}}+e^{-\mu L},
$$

where this expression is exact up to all powers of $L$, the only corrections being exponentially suppressed (which will not be important for our discussion, so we will ignore them). The functions $\rho_{n}(\{\theta\})$ are given by the determinant of the Jacobian of the transformation from integers, $\{I\}$, to rapidities, $\{\theta\}$,

$$
\begin{aligned}
J_{k l}\left(\theta_{1}, \ldots, \theta_{n}\right) & \equiv \frac{\partial}{\partial \theta_{l}} Q_{k}\left(\theta_{1}, \ldots, \theta_{n}\right), \\
\rho_{n}\left(\theta_{1}, \ldots, \theta_{n}\right) & \equiv\left|\operatorname{Det} J\left(\theta_{1}, \ldots, \theta_{n}\right)\right| .
\end{aligned}
$$

The finite-volume regularization of the form factor (35) is then

$$
f_{\vartheta}^{\mathcal{O}}(\theta+\pi \mathrm{i}, \theta+\kappa)=\lim _{L \rightarrow \infty} \frac{f^{\mathcal{O}}\left(\theta_{n}+\pi \mathrm{i}, \ldots, \theta_{1}+\pi \mathrm{i}, \theta_{1}+\kappa_{1}, \ldots, \theta_{n}-\kappa_{n}, \theta+\pi \mathrm{i}, \theta+\kappa\right)}{\rho_{n}\left(\theta_{1}, \ldots, \theta_{n}\right)},
$$

where, as derived in Appendix A, in the limit of a small particle-hole excitation,

$$
\kappa_{j}=-\frac{T_{L}^{\mathrm{dr}}\left(\theta_{j} \theta\right)}{L \rho_{L, s}\left(\theta_{j}\right)} \kappa
$$

Functions $T_{L}^{\mathrm{dr}}\left(\theta, \theta^{\prime}\right)$ and $\rho_{s, L}(\theta)$ are finite-volume versions of the standard thermodynamic functions $T^{\mathrm{dr}}\left(\theta, \theta^{\prime}\right)$ and $\rho_{s}(\theta)$. Their defining equations are given in Appendix A. The set of rapidities $\theta_{1}, \ldots, \theta_{n}$, with $n \sim L$ are chosen such that in the thermodynamic limit they are distributed according to occupation number $\vartheta(\theta)$,

$$
\lim _{L, n \rightarrow \infty} \sqrt{\rho_{n}\left(\theta_{1}, \ldots, \theta_{n}\right)}\left|I_{1}, \ldots, I_{n}\right\rangle=|\vartheta\rangle .
$$


Note that, in accordance with the definition of the thermodynamic form factors (24), the right hand side of 39 is normalized with respect to the background state.

The ordering of the rapidties in (39) follows the convention of the thermodynamic form factors, in which excitations appear after the background rapidities. In the limit $\kappa \rightarrow 0$ the ordering of (39) can be brought to the standard IQFT ordering in which $\theta+\pi \mathrm{i}$ appears first. To achieve this one has to scatter the corresponding particle with all other particles. In doing so the back-flow accumulates, however the back-flow is of order $\kappa$ and therefore does not contribute to the leading order when $\kappa \rightarrow 0$. Therefore

$$
f_{\vartheta}^{\mathcal{O}}(\theta+\pi \mathrm{i}, \theta+\kappa)=\lim _{L \rightarrow \infty} \frac{f^{\mathcal{O}}\left(\theta+\pi \mathrm{i}, \theta_{n}+\pi \mathrm{i}, \ldots, \theta_{1}+\pi \mathrm{i}, \theta_{1}+\kappa_{1}, \ldots, \theta_{n}-\kappa_{n}, \theta+\kappa\right)}{\rho_{n}\left(\theta_{1}, \ldots, \theta_{n}\right)} \times(1+\mathcal{O}(\kappa)) .
$$

This expression, together with the relation (40) between $\kappa_{j}$ and $\kappa$, is the starting point of our analysis.

To better understand expression (42), it is necessary to examine more closely the singularity structure of form factors. Following the annihilation-pole axiom [2, 3], form factors are singular whenever the rapidity of an incoming and outgoing particle approach each other. For an almost diagonal form factor (with all the particles in the incoming state approaching those in the outgoing state), we can express this singular structure as

$$
f^{\mathcal{O}}\left(\theta_{n}+\pi \mathrm{i}, \ldots, \theta_{1}+\pi \mathrm{i}, \theta_{1}+\kappa_{1}, \ldots, \theta_{n}+\kappa_{n}\right)=\frac{1}{\kappa_{1} \ldots \kappa_{n}} \sum_{\left\{i_{k}\right\}_{n}=1}^{n} a_{i_{1}, \ldots, i_{n}} \kappa_{i_{1}} \ldots \kappa_{i_{n}}
$$

for small $\left\{\kappa_{i}\right\}$, where the functions $a_{i_{1}, \ldots, i_{m}}$ are all finite and symmetric in the indices. The summation is

$$
\sum_{\left\{i_{k}\right\}_{n}=1}^{n}(\cdots)=\sum_{i_{1}=1}^{n} \cdots \sum_{i_{n}=1}^{n}(\cdots)
$$

It is convenient to extract certain finite values related to the diagonal form factors (43). First, one notices that the expression (43) is completely finite if all the regulators are taken to zero simultaneously, $\kappa_{i}=\kappa$, for all $i$. From this one can define the symmetric form factor, as

$$
\begin{aligned}
f_{s}^{\mathcal{O}}\left(\theta_{1}, \ldots, \theta_{n}\right) & \equiv \lim _{\kappa \rightarrow 0} f^{\mathcal{O}}\left(\theta_{n}+\pi \mathrm{i}, \ldots, \theta_{1}+\pi \mathrm{i}, \theta_{1}+\kappa, \ldots, \theta_{n}+\kappa\right) \\
& =\sum_{\left\{i_{k}\right\}_{n}=1}^{n} a_{i_{1}, \ldots, i_{n}} .
\end{aligned}
$$

Another useful quantity is the connected form factor, defined as the finite (non diverging) part of the form factor as any of the $\kappa_{i}$ is individually taken to zero. The connected form factor is then given by

$$
f_{c}^{\mathcal{O}}\left(\theta_{1}, \ldots, \theta_{n}\right) \equiv n ! a_{1, \ldots, n}
$$



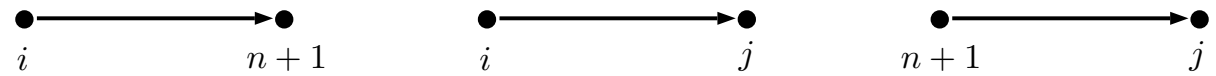

Figure 1: The three types of graphs contributing to $C^{1}$. We picture only the 2 connected nodes of each class, with all other nodes disconnected. The pictures correspond to the contributions $C^{1 a}, C^{1 b}$ and $C^{1 c}$, respectively.

Factor $n$ ! appears from summing over permutations of $\kappa_{i}$.

In [41] it was shown, that the almost diagonal form factor can be represented by connected form factors with different number of particles. The result is best understood in terms of sum over graphs which we now introduce following [41]. Let $G$ be a set of the directed graphs $G_{i}$ with $n$ vertices and with the following properties

1. $G_{i}$ is tree-like,

2. For each vertex there is at most one outgoing edge.

We denote $E_{j k}$ an edge going from $j$ to $k$. Then the diagonal form-factor is expressed as a sum over all graphs in $G$. With each node of the graph $G_{i}$ we associate a rapidity. Choice of graph $G_{i}$ divides the set of all the rapidities in two sets $\{\theta\}=\left\{\theta_{+}\right\} \cup\left\{\theta_{-}\right\}$, where we associate $\left\{\theta_{+}\right\}$to the nodes without outgoing arrow and $\left\{\theta_{-}\right\}$to the nodes with an outgoing arrow. Each graph contributes then

- a factor $f_{c}^{\mathcal{O}}\left(\left\{\theta_{+}\right\}\right)$

- for each edge $E_{j k}$, the form factor is multiplied by $2 \pi\left(\epsilon_{k} / \epsilon_{j}\right) T\left(\theta_{j}-\theta_{k}\right)$.

We now apply this general result of [41] to the particle-hole form factor (35) expressed as a thermodynamic limit of the finite volume form factor $(42)$. That is we consider $2(n+1)$ particle diagonal form factor with rapidities $\left\{\theta_{j}\right\}_{j=1}^{n}$ and $\theta$ and $\epsilon_{i}=\kappa_{i}$ with an exception that $\epsilon_{n+1}=\kappa$. For small $\kappa$ we have that $\kappa_{j}=\alpha_{j} \kappa$ where $\alpha_{i} \equiv T_{L}^{\mathrm{dr}}\left(\theta_{i}, \theta\right) / L \rho_{s, L}\left(\theta_{i}\right)$ for $i \in[1, n]$ and $\alpha_{n+1}=1$. This leads to the new rule, that for each edge $E_{j k}$ in the graph $G_{i}$ the form factor is multiplied by $2 \pi\left(\alpha_{k} / \alpha_{j}\right) T\left(\theta_{j}-\theta_{k}\right)$.

In the finite system this is the final result for the particle-hole form factor. The structure of terms contributing to the form factor changes in the thermodynamic limit. The reason for this is that all the factors $\alpha_{j}$ are of order $1 / L$ with an exception of $\alpha_{n+1}=1$. Therefore the contribution from the graphs were the $n+1$ node has ingoing edges are potentially more important in the thermodynamic limit. To analyse this carefully we first order the summation over graphs by the number of edges they contain. We denote $C^{m}$ contribution to the form-factor from graphs with $m$ edges. 
There is a single graph with $n+1$ nodes and without edges. Therefore

$$
C^{0} \equiv \frac{f_{c}^{\mathcal{O}}\left(\theta_{1}, \ldots, \theta_{n}, \theta\right)}{\rho_{n}\left(\theta_{1}, \ldots, \theta_{n}\right)}
$$

Graphs with a single edge can be further divided in 3 groups depending on whether the $(n+1)$-th node has an incoming edge, there is no edge connected to it, there is an outgoing edge. We call the 3 contributions $C^{1 a}, C^{1 b}$ and $C^{1 c}$ respectively. The graphs corresponding to each contribution are depicted in Fig. 1. We have

$$
\begin{aligned}
C^{1 a} & =2 \pi \sum_{i=1}^{n} \frac{f_{c}^{\mathcal{O}}\left(\{\theta\}_{n+1}, \hat{\theta}_{i}\right)}{\rho_{n}\left(\theta_{1}, \ldots, \theta_{n}\right)} \frac{1}{\alpha_{i}} T\left(\theta_{i}-\theta\right), \\
C^{1 b} & =2 \pi \sum_{\substack{i, j=1 \\
i \neq j}}^{n} \frac{f_{c}^{\mathcal{O}}\left(\{\theta\}_{n+1}, \hat{\theta}_{i}\right)}{\rho_{n}\left(\theta_{1}, \ldots, \theta_{n}\right)} \frac{\alpha_{j}}{\alpha_{i}} T\left(\theta_{i}-\theta_{j}\right), \\
C^{1 c} & =2 \pi \sum_{i=1}^{n} \frac{f_{c}^{\mathcal{O}}\left(\{\theta\}_{n+1}, \hat{\theta}_{n+1}\right)}{\rho_{n}\left(\theta_{1}, \ldots, \theta_{n}\right)} \alpha_{i} T\left(\theta-\theta_{i}\right),
\end{aligned}
$$

where we used that $\alpha_{n+1}=1$, and we have introduced the notation $\left(\{\theta\}_{n+1}, \hat{\theta}_{i}\right)$ to represent the set of $n+1$ rapidities, but where the rapidity $\theta_{i}$ has been removed. Contributions $C^{1 a}$ and $C^{1 b}$ are of order $L^{2}$, whereas $C^{1 c}$ is of order $L^{0}$ and can be neglected. In the thermodynamic limit, the leading contribution is thus

$$
C^{1}={ }_{\text {th }} 2 \pi \sum_{i=1}^{n} \frac{f_{c}^{\mathcal{O}}\left(\{\theta\}_{n+1}, \hat{\theta}_{i}\right)}{\rho_{n}\left(\theta_{1}, \ldots, \theta_{n}\right)} \frac{1}{\alpha_{i}}\left(T\left(\theta_{i}-\theta\right)+\sum_{\substack{j=1 \\ j \neq i}}^{n} \alpha_{j} T\left(\theta_{i}-\theta_{j}\right)\right)=2 \pi L \sum_{i=1}^{n} \frac{f_{c}^{\mathcal{O}}\left(\{\theta\}_{n+1}, \hat{\theta}_{i}\right) \rho_{s}\left(\theta_{i}\right)}{\rho_{n}\left(\theta_{1}, \ldots, \theta_{n}\right)}
$$

where we have introduced the equivalence symbol, $=_{\text {th }}$, to mean that the two expressions agree in the thermodynamic limit. In writing (49) we used the integral equation for the dressed scattering $T^{\mathrm{dr}}\left(\theta_{i}, \theta\right)$, Eq. 200 in its finite-volume version from Appendix A.

We can continue this way, computing the leading contributions $C^{m}$, coming from graphs with $m$ edges. We analyze in the detail the case of $m=2$ in the Appendix $B$. The result is

$$
C^{2}=_{\text {th }} \frac{1}{2} \sum_{\substack{i, j=1 \\ i \neq j}}^{n} \frac{f_{c}^{\mathcal{O}}\left(\{\theta\}_{n+1}, \hat{\theta}_{i}, \hat{\theta}_{j}\right) \tilde{\rho}_{n}\left(\theta_{i}, \theta_{j}\right)}{\rho_{n}\left(\theta_{1}, \ldots, \theta_{n}\right)}
$$

Similarly one can continue calculating $C^{m}$ for each value of $m$, from which we can extrapolate the general expression

$$
\lim _{\kappa \rightarrow 0} f_{\vartheta}^{\mathcal{O}}(\theta+\pi \mathrm{i}, \theta+\kappa)=\lim _{L, n \rightarrow \infty} \sum_{m=1}^{n} C^{m}=\lim _{L, n \rightarrow \infty} \sum_{\left\{\theta_{+}\right\} \cup\left\{\theta_{-}\right\}=\{\theta\}} \frac{f_{c}^{\mathcal{O}}\left(\left\{\theta_{+}\right\}, \theta\right) \tilde{\rho}_{n}\left(\left\{\theta_{-}\right\}\right)}{\rho_{n}\left(\left\{\theta_{-}\right\} \cup\left\{\theta_{+}\right\}\right)},
$$


where we are summing over all the possible bipartitions of the set of rapidities $\{\theta\}=\left\{\theta_{1}, \ldots, \theta_{n}\right\}=$ $\left\{\theta_{-}\right\} \cup\left\{\theta_{+}\right\}$. We have also $\tilde{\rho}_{n}\left(\left\{\theta_{-}\right\}\right)$as the determinant of the sub-matrix which is defined in terms of the Jacobian $J_{k l}$, by selecting only the rows and colums with the indices $k, l$ corresponding to particles in the subset $\left\{\theta_{-}\right\}$.

We want to further evaluate the expression (51), term by term in the thermodynamic limit. This calculation will be similar to the derivation of the Leclair-Mussardo formula shown in Ref. [42]. To do this, it is convenient to start with the term corresponding to $\left\{\theta_{+}\right\}=\emptyset$ and $\left\{\theta_{-}\right\}=\{\theta\}$. Then we will evaluate the next term where $\left\{\theta_{+}\right\}$contains only one particle, and so on. We can thus express (51) as a series

$$
\lim _{\kappa \rightarrow 0} f_{\vartheta}^{\mathcal{O}}(\theta+\pi \mathrm{i}, \theta+\kappa)=D^{0}+D^{1}+D^{2}+\ldots
$$

where $D^{i}$ are the contributions in 51 where $\left\{\theta_{+}\right\}$contains $i$ particles. We will assume the sum over bipartitions and the thermodynamic limit can be exchanged, such that we will take the thermodynamic limit of each term individually.

First, we consider the term $D^{0}$, which corresponds simply to the form factor

$$
D^{0}=f_{c}^{\mathcal{O}}(\theta)
$$

For the next term, $D^{1}$, we consider $\left\{\theta_{-}\right\}$with one particle, and sum over all these one particle contributions, we find

$$
D^{1}=\int \frac{d \theta_{1}}{2 \pi} \vartheta\left(\theta_{1}\right) f_{c}^{\mathcal{O}}\left(\theta_{1}, \theta\right)
$$

where we used that 42

$$
\frac{\tilde{\rho}_{n}\left(\theta_{1}, \ldots, \theta_{i-1}, \theta_{i+1}, \ldots, \theta_{n}\right)}{\rho_{n}\left(\theta_{1}, \ldots, \theta_{n}\right)}=\frac{1}{2 \pi L \rho_{s}\left(\theta_{i}\right)},
$$

and expressed the discrete sum over particles as an integral over rapidities,

$$
\lim _{L, n \rightarrow \infty} \sum_{i} f\left(\theta_{i}\right)=L \int d \theta \rho_{p}(\theta) f(\theta) .
$$

It is then straightforward to continue evaluating, term by term, contributions with more particles in $\left\{\theta_{+}\right\}$. This produces the series,

$$
\lim _{\kappa \rightarrow 0} f_{\vartheta}^{\mathcal{O}}(\theta+\pi \mathrm{i}, \theta+\kappa)=2 \pi \rho_{s}(\theta) V^{\mathcal{O}}(\theta)=\sum_{k=0}^{\infty} \frac{1}{k !} \int_{S^{\times k}} \prod_{j=1}^{k}\left(\frac{d \theta_{j}}{2 \pi} \vartheta\left(\theta_{j}\right)\right) f_{c}^{\mathcal{O}}\left(\theta_{1}, \ldots, \theta_{k}, \theta\right) .
$$


This formula is the main result of this section. As we will see in Section 6, it reproduces the result for correlators at the Eulerian scale, derived from the GHD formalism [13], while we reinstate that absolutely no knowledge or assumptions from GHD were used in our derivation.

We note that the result (57) can be interpreted as one additional axiom that complements those introduced in [1], which comprise the thermodynamic bootstrap program. In fact from the previous known axioms derived in [1], it is possible to compute particle-hole form factors only up to some ambiguous pre-factor.

In [1, based on the available knowledge at the time, particle-hole form factors for the sinh-Gordon model were computed, and the simplest possible pre-factor (a constant, independent of particle rapidities) was assumed. In light of our new result, (57), we see that this minimal choice was too simple, but our new result can be used to fix this ambiguous pre-factor.

We point out the similarity of our result (57) to dressed form factors in the crossed channel previously considered in [43]. We recall that due to Lorentz invariance, the partition function of a $(1+1)-d$ QFT at finite temperature and infinite volume is equivalent to that of a finite-volume theory at zero temperature. The form factors studied in [43] concern particle excitations of the finite-volume Hamiltonian at zero temperature, while our result involves the infinite-volume particle excitations, dressed by a finite temperature (or GGE background). It seems a very intriguing coincidence that the dressing procedure for form factors in both channels seems to match, up to some imaginary shifts in the particle rapidities, while this is not expected to be the case, a priori. This connection is a subject that merits a deeper study in the future.

\section{$5 \quad$ Two and more particle-hole pairs}

Computing the low-momentum limit of form factors with a higher number of pairs is now straightforward and the formula reduces to the single particle-hole pair form factors through the annihilation pole axiom (30).

We consider the simplest generalization, to the case of two particle-hole pairs at low momentum. For a generic form factor of an operator, $\mathcal{O}$, in the presence of a background, the annihilation pole axiom requires

$$
\begin{aligned}
& \lim _{\kappa_{1,2} \rightarrow 0} f_{\vartheta}^{\mathcal{O}}\left(\theta_{1}+\pi \mathrm{i}+\kappa_{1}, \theta_{1}, \theta_{2}+\pi \mathrm{i}+\kappa_{2}, \theta_{2}\right) \\
& \quad=\lim _{\kappa_{1,2} \rightarrow 0} \frac{\mathrm{i}}{\kappa_{1}}\left[1-R\left(\theta_{1} \mid \theta_{2}+\pi \mathrm{i}+\kappa_{2}, \theta_{2}\right) S\left(\theta_{1}-\theta_{2}-\pi \mathrm{i}-\kappa_{2}\right) S\left(\theta_{1}-\theta_{2}\right)\right] f_{\vartheta}^{\mathcal{O}}\left(\theta_{2}+\pi \mathrm{i}+\kappa_{2}, \theta_{2}\right),(58)
\end{aligned}
$$


with function $R$ defined in (33). The factor in brackets in the right-hand side vanishes in the $\kappa_{2} \rightarrow 0$ limit. The leading contribution to the 2-particle-hole pairs form factor at this point comes at order $\mathcal{O}\left(\kappa_{2} / \kappa_{1}\right)$, which is finite, as long as $\kappa_{2} \sim \kappa_{1}$ as we take the $\kappa_{1,2} \rightarrow 0$ limit. We find

$$
\lim _{\kappa_{1,2} \rightarrow 0} f_{\vartheta}^{\mathcal{O}}\left(\theta_{1}+\pi \mathrm{i}+\kappa_{1}, \theta_{1}, \theta_{2}+\pi \mathrm{i}+\kappa_{2}, \theta_{2}\right)=\lim _{\kappa_{1,2} \rightarrow 0} 2 \pi \frac{\kappa_{2}}{\kappa_{1}} \frac{\partial F\left(\theta_{1} \mid \theta_{2}\right)}{\partial \theta_{2}} f_{\vartheta}^{\mathcal{O}}\left(\theta_{2}+\pi \mathrm{i}+\kappa_{2}, \theta_{2}\right) .
$$

The derivative of the back-flow is related to the dressed scattering phase shift (16).

$$
\frac{\partial F\left(\theta_{1} \mid \theta_{2}\right)}{\partial \theta_{2}}=-T^{\mathrm{dr}}\left(\theta_{1}, \theta_{2}\right)
$$

Hence

$$
\lim _{\kappa_{1,2} \rightarrow 0} f_{\vartheta}^{\mathcal{O}}\left(\theta_{1}+\pi \mathrm{i}+\kappa_{1}, \theta_{1}, \theta_{2}+\pi \mathrm{i}+\kappa_{2}, \theta_{2}\right)=-2 \pi \lim _{\kappa_{1,2} \rightarrow 0} \frac{\kappa_{2}}{\kappa_{1}} T^{\mathrm{dr}}\left(\theta_{1}, \theta_{2}\right) f_{\vartheta}^{\mathcal{O}}\left(\theta_{2}+\pi \mathrm{i}+\kappa_{2}, \theta_{2}\right) .
$$

We can evaluate further the structure of poles of the 2 particle-hole-pair form factor, by considering the different permutations of particles and holes that may annihilate with each other. Through the annihilation-pole axiom, we can fully determine the non-analytic part of the form factors as a function of 4 rapidities. We find

$$
f_{\vartheta}^{\mathcal{O}}\left(\theta_{1}, \theta_{2}, \theta_{3}, \theta_{4}\right)=-2 \pi \sum_{\sigma \in P_{4}} S_{\sigma}\left(\theta_{1}, \ldots, \theta_{4}\right) \frac{\theta_{\sigma_{3}}-\theta_{\sigma_{4}}-i \pi}{\theta_{\sigma_{1}}-\theta_{\sigma_{1}}-i \pi} T^{\mathrm{dr}}\left(\theta_{\sigma_{2}}, \theta_{\sigma_{4}}\right) f_{\vartheta}^{\mathcal{O}}\left(\theta_{\sigma_{3}}, \theta_{\sigma_{4}}\right)+\text { regular }
$$

where $S_{\sigma}\left(\theta_{1}, \ldots, \theta_{4}\right)$ is a product over the $S$-matrices obtained from representing permutation $\sigma$ as a product of adjacent transpositions and then writing the $S$-matrix for every transposition. For example, for permutation $\sigma=(1423)=(23)(34)$,

$$
S_{(1423)}\left(\theta_{1}, \ldots, \theta_{4}\right)=S\left(\theta_{2}-\theta_{4}\right) S\left(\theta_{3}-\theta_{4}\right)
$$

The pole structure of the form factor 62 agrees with the conjectured form factors in [30]. It has been shown in [30, that if the 2-particle-hole-pair form factors of current density operators are of the form (62), this leads to a diffusion matrix of the form (9).

The structure carries on for higher number of particle-hole pairs. For example, for 3 particle-hole pairs, taking the limit first $\kappa_{1}$, then with $\kappa_{2}$ and finally with $\kappa_{3}$ we find

$$
\begin{aligned}
& \lim _{\kappa_{1,2,3} \rightarrow 0} f_{\vartheta}^{\mathcal{O}}\left(\theta_{1}+\pi \mathrm{i}+\kappa_{1}, \theta_{1}, \theta_{2}+\pi \mathrm{i}+\kappa_{2}, \theta_{2}, \theta_{3}+\pi \mathrm{i}+\kappa_{3}, \theta_{3}\right) \\
& \quad=\lim _{\kappa_{1,2,3} \rightarrow 0}(2 \pi)^{2}\left[\frac{\kappa_{3}}{\kappa_{1}} T^{\mathrm{dr}}\left(\theta_{1}, \theta_{2}\right) T^{\mathrm{dr}}\left(\theta_{2}, \theta_{3}\right)+\frac{\kappa_{3}^{2}}{\kappa_{1} \kappa_{2}} T^{\mathrm{dr}}\left(\theta_{1}, \theta_{3}\right) T^{\mathrm{dr}}\left(\theta_{2}, \theta_{3}\right)\right] f_{\vartheta}^{\mathcal{O}}\left(\theta_{3}+\pi \mathrm{i}+\kappa_{3}, \theta_{3}\right),(64)
\end{aligned}
$$

Considering the limit in different orders and mixing the particle and holes pairs we can capture the whole singularity structure of the form factor in a formula generalizing 62. 


\section{Euler-scale correlators from particle-hole form factors}

In this section we will show how our results for the single particle-hole pair form factors from Section 4 yield the Euler-scale correlation functions predicted from the GHD formalism in Ref. [13]. We are interested in the Euler-scale correlator

$$
C^{\mathcal{O}_{1} \mathcal{O}_{2}}(\xi, t) \equiv \lim _{t \rightarrow \infty} \frac{\left\langle\vartheta\left|\mathcal{O}_{1}(\xi t, t) \mathcal{O}_{2}(0,0)\right| \vartheta\right\rangle}{\langle\vartheta \mid \vartheta\rangle}-\frac{\left\langle\vartheta\left|\mathcal{O}_{1}\right| \vartheta\right\rangle}{\langle\vartheta \mid \vartheta\rangle} \frac{\left\langle\vartheta\left|\mathcal{O}_{2}\right| \vartheta\right\rangle}{\langle\vartheta \mid \vartheta\rangle}
$$

Within the TBP formalism, the general expression for the two-point function is given by Eq. 25). Taking the $t \rightarrow \infty$ limit, however, means that we can focus only on low-energy excitations, as intermediate states with a high number of particles and holes are suppressed at long times/distances. At the Euler scale, we therefore consider only the lowest-lying excitation: a single particle-hole pair, whose contribution is

$$
\begin{aligned}
C^{\mathcal{O}_{1} \mathcal{O}_{2}}(\xi, t)= & \lim _{t \rightarrow \infty} \int \frac{d \theta_{1}}{2 \pi} \frac{d \theta_{2}}{2 \pi} \vartheta\left(\theta_{1}\right)\left(1-\vartheta\left(\theta_{2}\right)\right) f_{\vartheta}^{\mathcal{O}_{1}}\left(\theta_{1}, \theta_{2}+\pi \mathrm{i}\right)\left(f_{\vartheta}^{\mathcal{O}_{2}}\left(\theta_{1}, \theta_{2}+\pi \mathrm{i}\right)\right)^{*} \\
& \times \exp \left\{\mathrm{i} t\left[\xi\left(k\left(\theta_{1}\right)-k\left(\theta_{2}\right)\right)-\left(\omega\left(\theta_{1}\right)-\omega\left(\theta_{2}\right)\right)\right]\right\} .
\end{aligned}
$$

In the large $t$ limit, this integral can be readily computed using a two-dimensional stationary phase approximation. Defining the two-dimensional vector $\vec{\theta}=\left(\theta_{1}, \theta_{2}\right)$, and the functions in this space

$$
\begin{aligned}
& g(\vec{\theta})=\frac{\vartheta\left(\theta_{1}\right)\left(1-\vartheta\left(\theta_{2}\right)\right)}{(2 \pi)^{2}} f_{\vartheta}^{\mathcal{O}_{1}}\left(\theta_{1}, \theta_{2}+\pi \mathrm{i}\right)\left(f_{\vartheta}^{\mathcal{O}_{2}}\left(\theta_{1}, \theta_{2}+\pi \mathrm{i}\right)\right)^{*}, \\
& h(\vec{\theta})=\xi\left[k\left(\theta_{1}\right)-k\left(\theta_{2}\right)\right]-\omega\left(\theta_{1}\right)+\omega\left(\theta_{2}\right),
\end{aligned}
$$

the stationary phase approximation yields

$$
C^{\mathcal{O}_{1} \mathcal{O}_{2}}(\xi, t)=g\left(\overrightarrow{\theta_{0}}\right)\left|\operatorname{det} H\left[h\left(\overrightarrow{\theta_{0}}\right)\right]\right|^{-1 / 2} \exp \left[\mathrm{i} t h\left(\vec{\theta}_{0}\right)+\mathrm{i} \frac{1}{4} \sigma_{H}\right] \frac{2 \pi}{t},
$$

where $H[h(\vec{\theta})]$ is the Hessian of the function $h(\vec{\theta})$, i.e. the two-by-two matrix

$$
H_{i j}[h(\vec{\theta})]=\frac{\partial^{2} h(\vec{\theta})}{\partial \theta_{i} \partial \theta_{j}}
$$

$\vec{\theta}_{0}$ are the set of critical points that satisfy $\left.\nabla h(\theta)\right|_{\vec{\theta}=\vec{\theta}_{0}}=0$ and $\sigma_{H}$ is the signature of the Hessian (number of positive eigenvalues minus number of negative eigenvalues) evaluated at $\vec{\theta}=\vec{\theta}_{0}$.

The Hessian can then be expressed as the matrix,

$$
H[h(\vec{\theta})]=\left(\begin{array}{cc}
k^{\prime \prime}\left(\theta_{1}\right)\left(\xi-v^{\mathrm{eff}}\left(\theta_{1}\right)\right)-k^{\prime}\left(\theta_{1}\right)\left(v^{\mathrm{eff}}\right)^{\prime}\left(\theta_{1}\right) & 0 \\
0 & -k^{\prime \prime}\left(\theta_{2}\right)\left(\xi-v^{\mathrm{eff}}\left(\theta_{2}\right)\right)+k^{\prime}\left(\theta_{2}\right)\left(v^{\mathrm{eff}}\right)^{\prime}\left(\theta_{2}\right)
\end{array}\right)
$$


The critical points are also easily computed, giving $\vec{\theta}_{0}=\left(\theta_{*}(\xi), \theta_{*}(\xi)\right)$, where we recall from Section 2 the definition $v^{\text {eff }}\left[\theta_{*}(\xi)\right]=\xi$. With this information, it is easy to verify that $\sigma_{H}=0$, and that

$$
\left|\operatorname{det} H\left[h\left(\vec{\theta}_{0}\right)\right]\right|^{1 / 2}=k^{\prime}\left(\theta_{*}\right)\left(v^{\text {eff }}\right)^{\prime}\left(\theta_{*}\right)=2 \pi \rho_{s}\left(\theta_{*}\right)\left(v^{\text {eff }}\right)^{\prime}\left(\theta_{*}\right) .
$$

At the critical points $\vec{\theta}_{0}$, both rapidities are equal, $\theta_{1}=\theta_{2}=\theta_{*}(\xi)$, therefore we can directly use the results for the particle-hole form factors from Eq. (57). Lastly, to match more closely the notation of 13 , we notice we can write

$$
g\left(\vec{\theta}_{0}\right)=(2 \pi)^{2} \rho_{s}\left(\theta_{*}\right) \rho_{p}\left(\theta_{*}\right)\left(1-\vartheta\left(\theta_{*}\right)\right) V^{\mathcal{O}_{1}}\left(\theta_{*}\right) V^{\mathcal{O}_{2}}\left(\theta_{*}\right)
$$

Putting all these ingredients together into $(68)$ we finally arrive at the correlator

$$
C^{\mathcal{O}_{1} \mathcal{O}_{2}}(\xi, t)=t^{-1} \sum_{\theta \in \theta_{*}(\xi)} \frac{\rho_{p}(\theta)(1-\vartheta(\theta))}{\left|\left(v^{\mathrm{eff}}\right)^{\prime}(\theta)\right|} V^{\mathcal{O}_{1}}(\theta) V^{\mathcal{O}_{2}}(\theta)
$$

with $V^{\mathcal{O}}(\theta)$ given by Eq. (57). We have thus reproduced precisely the GHD prediction [13] for the Euler-scale correlation function (5) by performing a form factor expansion, and keeping only the leading contribution, from one particle-hole pair excitations.

We point out that we could also use the two-particle-hole pairs form factors, from Eq. 62 to derive the diffusion matrix (9). Since this calculation has already been shown in Ref. [30, we will not reproduce it here, but refer the reader to the original paper.

\section{Conclusions}

We have shown that the predictions of the Thermodynamic Bootstrap Program in the hydrodynamic regime agree with those of Generalized Hydrodynamics.

We have shown that the form factor for a generic local operator in an integrable QFT, with one particle-hole pair on top of a thermodynamic background, can be computed, in the limit when the two rapidites approach each other, in terms of a LeClair-Mussardo-like expansion over connected form factors. These form factors can be used to calculate two-point functions of operators, and we have shown that to compute Euler-scale correlators, it is sufficient to include the one-particle-hole form factors. We have shown that the correlation function thus derived exactly matches the GHD prediction from Ref. [13.

Additionally, we computed the pole structure of form factors with 2 particle-hole pairs, corresponding to the Navier-Stokes GHD regime, through the TBP annihilation pole axiom, accounting for dressing by 
the thermodynamic background. Our result agrees with the conjectured 2 particle-hole pairs form factor structure that leads to appropriate diffusive transport.

An interesting question for the future is about establishing a systematic gradient expansion of the GHD. For this, the necessary ingredients are higher particle-hole pairs form factors of conserved densities and currents. We have shown that the Thermodynamic Bootstrap program automatically generates such form factors and might be of value in further developing GHD in that direction.

The results of this work also serve as a non-trivial check of the correctness of the Thermodynamic Bootstrap Program. In particular, the results for the 2-particle-hole pair form factors suggest that the annihilation-pole axiom, dressed by the thermodynamic background, as proposed in 1, is indeed correct. It would be interesting to further develop computations within the TBP, to provide other non-trivial checks against the wealth of results from the GHD literature.

\section{Acknowledgments}

The authors thank Jacopo De Nardis and Benjamin Doyon for valuable insights and comments on the early version of this work. ACC acknowledges the support from the European Research Council under ERC Advanced grant 743032 DYNAMINT, as well as support from the European Union's Horizon 2020 research and innovation programme under the Marie Skłodowska-Curie grant agreement 75009 at early stages of this project. MP acknowledges the support from the National Science Centre under SONATA grant 2018/31/D/ST3/03588.

\section{A Back-flow of the rapidities in the presence of a small particle-hole excitation}

In this appendix we consider the change to the rapidities of the background state induced by a presence of a particle-hole excitation of a small momentum. The rapidities of the background state in a finite system fulfil the Bethe equations

$$
m L \sinh \theta_{j}=2 \pi I_{j}-\sum_{\substack{k=1 \\ k \neq j}}^{n} \delta\left(\theta_{j}-\theta_{k}\right), \quad j=1, \ldots, n .
$$


Creating a particle-hole excitations amounts to adding a particle $\bar{\theta}$ and anti-particle $\theta$. Because of the coupled nature of the Bethe equations this leads to a change in all the other rapidities as well. We denote the set of modified rapidities by $\{\bar{\theta}\}$ and consider a difference between the Bethe equations for rapidities in the excited state and the background state

$$
m L\left(\sinh \bar{\theta}_{j}-\sinh \theta_{j}\right)=-\sum_{\substack{k=1 \\ k \neq j}}^{n}\left(\delta\left(\bar{\theta}_{j}-\bar{\theta}_{k}\right)-\delta\left(\theta_{j}-\theta_{k}\right)\right)-\delta\left(\bar{\theta}_{j}-\bar{\theta}\right)+\delta\left(\bar{\theta}_{j}-\theta\right), \quad j=1, \ldots, n .
$$

A particle-hole excitation is small if $\bar{\theta}=\theta+\kappa$ with small parameter $\kappa$. As a consequence, the difference $\bar{\theta}_{j}-\theta_{j}$ is also small. Expanding the equality in this difference we find in the leading order

$$
m L\left(\bar{\theta}_{j}-\theta_{j}\right) \cosh \theta_{j}=-2 \pi \sum_{\substack{k=1 \\ k \neq j}}^{n}\left(\bar{\theta}_{j}-\theta_{j}-\bar{\theta}_{k}+\theta_{k}\right) T\left(\theta_{j}-\theta_{k}\right)-2 \pi \kappa T\left(\theta_{j}-\theta_{n}\right) .
$$

Reorganizing the sum leads to

$$
L\left(\bar{\theta}_{j}-\theta_{j}\right)\left(\frac{m \cosh \theta_{j}}{2 \pi}+\frac{1}{L} \sum_{\substack{k=1 \\ k \neq j}}^{n-1} \phi\left(\theta_{j}-\theta_{k}\right)\right)=\sum_{\substack{k=1 \\ k \neq j}}^{n-1}\left(\bar{\theta}_{k}-\theta_{k}\right) T\left(\theta_{j}-\theta_{k}\right)-\kappa T\left(\theta_{j}-\theta_{n}\right) .
$$

We define now two functions

$$
\begin{aligned}
\rho_{L}(\theta) & =\frac{m \cosh \theta}{2 \pi}+\frac{1}{L} \sum_{\substack{k=1 \\
k \neq j}}^{n} T\left(\theta-\theta_{k}\right), \\
T_{L}^{\mathrm{dr}}\left(\theta_{j}, \theta\right) & =-L\left(\bar{\theta}_{j}-\theta_{j}\right) \rho_{L}\left(\theta_{j}\right) / \kappa .
\end{aligned}
$$

In terms of them we find

$$
T_{L}^{\mathrm{dr}}\left(\theta_{j}, \theta\right)=T\left(\theta_{j}-\theta_{n}\right)+\frac{1}{L} \sum_{\substack{k=1 \\ k \neq j}}^{n} \rho_{L}\left(\theta_{k}\right) T\left(\theta_{j}-\theta_{k}\right) T_{L}^{\mathrm{dr}}\left(\theta_{k}, \theta\right)
$$

In the thermodynamic limit, $\rho_{L}(\theta)$ and $T_{L}^{\mathrm{dr}}\left(\theta_{j}, \theta\right)$ approach the standard TBA functions $\rho_{s}(\theta)$ and the dressed differential scattering kernel $T^{\mathrm{dr}}\left(\theta_{j}, \theta\right)$.

\section{B Computation of the term $C^{2}$}

We consider the contributions from graphs containing two edges. The three types of the leading contributions are shown in Fig. 2. They are all of order $L^{4}$. The contributions from graphs with an edge 

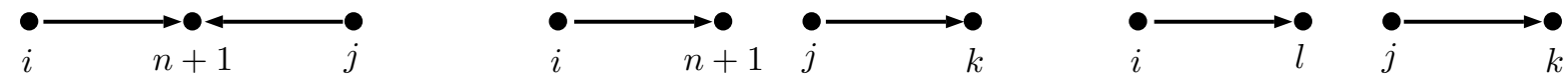

Figure 2: The three types of graphs contributing to $C^{2}$ (at leading order at large $L$ ), with all the disconnected nodes not pictured. The pictures correspond to the contributions $C^{2 a}, C^{2 b}$ and $C^{2 c}$, respectively.

originating at the $n+1$-th node are suppressed by $L^{2}$. In summing over graphs we need to be careful to include only tree-like graphs. It is convenient to actually sum over all the graphs and at the end subtract contributions from graphs containing loops. The 3 leading contributions are

$$
\begin{aligned}
C^{2 a} & =\frac{(2 \pi)^{2}}{2} \sum_{\substack{i, j=1 \\
i \neq j}}^{n} \frac{f_{c}^{\mathcal{O}}\left(\hat{\theta}_{i}, \hat{\theta}_{j}\right)}{\rho_{n}\left(\theta_{1}, \ldots, \theta_{n}\right)} \frac{1}{\alpha_{i} \alpha_{j}} T\left(\theta_{i}-\theta\right) T\left(\theta_{j}-\theta\right), \\
C^{2 b} & =(2 \pi)^{2} \sum_{\substack{i, j, k=1 \\
i \neq j, k \neq j}}^{n} \frac{f_{c}^{\mathcal{O}}\left(\hat{\theta}_{i}, \hat{\theta}_{j}\right)}{\rho_{n}\left(\theta_{1}, \ldots, \theta_{n}\right)} \frac{\alpha_{k}}{\alpha_{i} \alpha_{j}} T\left(\theta_{i}-\theta\right) T\left(\theta_{j}-\theta_{k}\right), \\
C^{2 c} & =\frac{(2 \pi)^{2}}{2} \sum_{\substack{i, j, k, l=1 \\
i \neq j, k \neq j, l \neq i}}^{n} \frac{f_{c}^{\mathcal{O}}\left(\hat{\theta}_{i}, \hat{\theta}_{j}\right)}{\rho_{n}\left(\theta_{1}, \ldots, \theta_{n}\right)} \frac{\alpha_{k} \alpha_{l}}{\alpha_{i} \alpha_{j}} T\left(\theta_{i}-\theta_{l}\right) T\left(\theta_{j}-\theta_{k}\right) .
\end{aligned}
$$

There is only one way the loop can be formed with two edges, this corresponds to contribution $C^{2 c}$ with $l=j$ and $k=i$. Therefore, the additional contributions that we need to subtract are

$$
\bar{C}^{2}=\frac{(2 \pi)^{2}}{2} \sum_{\substack{i, j=1 \\ i \neq j}}^{n} \frac{f_{c}^{\mathcal{O}}\left(\hat{\theta}_{i}, \hat{\theta}_{j}\right)}{\rho_{n}\left(\theta_{1}, \ldots, \theta_{n}\right)} T^{2}\left(\theta_{i}-\theta_{j}\right)
$$

We analyze the resulting contributions in few steps. First let us consider the contributions from the first two classes of graphs. We find

$$
\begin{aligned}
C^{2 a}+\frac{1}{2} C^{2 b} & =\frac{(2 \pi)^{2}}{2} \sum_{\substack{i, j=1 \\
i \neq j}}^{n} \frac{f_{c}^{\mathcal{O}}\left(\hat{\theta}_{i}, \hat{\theta}_{j}\right)}{\rho_{n}\left(\theta_{1}, \ldots, \theta_{n}\right)} \frac{T\left(\theta_{i}-\theta\right)}{\alpha_{i} \alpha_{j}}\left(T\left(\theta_{j}-\theta\right)+\sum_{\substack{k=1 \\
k \neq j}}^{n} \alpha_{k} T\left(\theta_{j}-\theta_{k}\right)\right) \\
& =\frac{(2 \pi)^{2}}{2} L \sum_{\substack{i, j=1 \\
i \neq j}}^{n} \frac{f_{c}^{\mathcal{O}}\left(\hat{\theta}_{i}, \hat{\theta}_{j}\right)}{\rho_{n}\left(\theta_{1}, \ldots, \theta_{n}\right)} \frac{\rho_{L}\left(\theta_{j}\right) T\left(\theta_{i}-\theta\right)}{\alpha_{i}}
\end{aligned}
$$

In a similar fashion, looking at the second and third class together, we find

$$
\frac{1}{2} C^{2 b}+C^{2 c}=\frac{(2 \pi)^{2} L}{2} \sum_{\substack{i, j=1 \\ i \neq j}}^{n} \frac{f_{c}^{\mathcal{O}}\left(\hat{\theta}_{i}, \hat{\theta}_{j}\right)}{\rho_{n}\left(\theta_{1}, \ldots, \theta_{n}\right)} \frac{\rho_{L}\left(\theta_{j}\right)}{\alpha_{i}} \sum_{\substack{k=1 \\ k \neq i}}^{n} \alpha_{k} T\left(\theta_{i}-\theta_{k}\right) .
$$


The total contribution $C^{2}$, with the extra terms subtracted, is then

$$
C^{2}=\frac{(2 \pi L)^{2}}{2} \sum_{\substack{i, j=1 \\ i \neq j}}^{n} \frac{f_{c}^{\mathcal{O}}\left(\hat{\theta}_{i}, \hat{\theta}_{j}\right)}{\rho_{n}\left(\theta_{1}, \ldots, \theta_{n}\right)}\left(\rho_{L}\left(\theta_{i}\right) \rho_{L}\left(\theta_{j}\right)-\frac{1}{L^{2}} T^{2}\left(\theta_{i}-\theta_{j}\right)\right)
$$

Consider now a determinant of the sub-matrix defined in terms of the Jacobian $J_{k l}$ by selecting only the $i$-th and $j$-th columns and rows

$$
\tilde{\rho}_{2}\left(\theta_{i}, \theta_{j}\right)=(2 \pi L)^{2} \operatorname{det}\left(\begin{array}{cc}
\rho_{L}\left(\theta_{i}\right) & -\frac{1}{L} T\left(\theta_{i}-\theta_{j}\right) \\
-\frac{1}{L} T\left(\theta_{i}-\theta_{j}\right) & \rho_{L}\left(\theta_{j}\right)
\end{array}\right)
$$

Evaluating the determinant we get

$$
\tilde{\rho}_{2}\left(\theta_{i}, \theta_{j}\right)=(2 \pi L)^{2}\left(\rho_{L}\left(\theta_{i}\right) \rho_{L}\left(\theta_{j}\right)-\frac{1}{L^{2}} T^{2}\left(\theta_{i}-\theta_{j}\right)\right)
$$

Therefore

$$
C^{2}=\frac{1}{2} \sum_{\substack{i, j=1 \\ i \neq j}}^{n} \frac{f_{c}^{\mathcal{O}}\left(\hat{\theta}_{i}, \hat{\theta}_{j}\right) \tilde{\rho}_{2}\left(\theta_{i}, \theta_{j}\right)}{\rho_{n}\left(\theta_{1}, \ldots, \theta_{n}\right)}
$$

\section{References}

[1] A. Cortés Cubero and M. Panfil, Thermodynamic bootstrap program for integrable QFT's: form factors and correlation functions at finite energy density, Journal of High Energy Physics 2019 (Jan, 2019) 104, 1809.02044.

[2] M. Karowski and P. Weisz, Exact form factors in $(1+1)$-dimensional field theoretic models with soliton behaviour, Nuclear Physics B 139 (1978) 455 - 476.

[3] F. A. Smirnov, Form Factors in Completely Integrable Models of Quantum Field Theory. World Scientific, 1992, 10.1142/1115.

[4] G. Mussardo, Statistical field theory : an introduction to exactly solved models in statistical physics. Oxford ; New York : Oxford University Press, 2009.

[5] A. Zamolodchikov, Thermodynamic Bethe ansatz in relativistic models: Scaling 3-state potts and Lee-Yang models, Nuclear Physics B 342 (1990) 695-720. 
[6] J.-S. Caux and R. M. Konik, Constructing the generalized Gibbs ensemble after a quantum quench, Phys. Rev. Lett. 109 (Oct, 2012) 175301, 1203.0901.

[7] J. Mossel and J.-S. Caux, Generalized TBA and generalized Gibbs, Journal of Physics A Mathematical General 45 (Jun, 2012) 255001, 1203.1305.

[8] J.-S. Caux and F. H. L. Essler, Time evolution of local observables after quenching to an integrable model, Phys. Rev. Lett. 110 (Jun, 2013) 257203, 1301.3806.

[9] B. Bertini, M. Collura, J. De Nardis and M. Fagotti, Transport in out-of-equilibrium XXZ chains: Exact profiles of charges and currents, Phys. Rev. Lett. 117 (Nov, 2016) 207201, 1605.09790.

[10] O. A. Castro-Alvaredo, B. Doyon and T. Yoshimura, Emergent hydrodynamics in integrable quantum systems out of equilibrium, Phys. Rev. X 6 (2016) 041065.

[11] B. Doyon, J. Dubail, R. Konik and T. Yoshimura, Large-scale description of interacting one-dimensional Bose gases: Generalized hydrodynamics supersedes conventional hydrodynamics, Phys. Rev. Lett. 119 (Nov, 2017) 195301, 1704.04151.

[12] D. Bernard and B. Doyon, Diffusion and signatures of localization in stochastic conformal field theory, Phys. Rev. Lett. 119 (Sep, 2017) 110201, 1612.05956.

[13] B. Doyon, Exact large-scale correlations in integrable systems out of equilibrium, SciPost Physics 5 (Nov, 2018) 054, 1711.04568.

[14] B. Doyon, H. Spohn and T. Yoshimura, A geometric viewpoint on generalized hydrodynamics, Nuclear Physics B 926 (Jan, 2018) 570-583, 1704.04409.

[15] B. Doyon, T. Yoshimura and J.-S. Caux, Soliton gases and generalized hydrodynamics, Phys. Rev. Lett. 120 (Jan, 2018) 045301, 1704.05482.

[16] A. Bastianello, B. Doyon, G. Watts and T. Yoshimura, Generalized hydrodynamics of classical integrable field theory: the sinh-gordon model, SciPost Physics 4 (Jun, 2018) 045, 1712.05687.

[17] B. Doyon, Generalised hydrodynamics of the classical Toda system, arXiv e-prints (Feb, 2019) arXiv:1902.07624, 1902.07624.

[18] L. Piroli, J. De Nardis, M. Collura, B. Bertini and M. Fagotti, Transport in out-of-equilibrium XXZ chains: Nonballistic behavior and correlation functions, Physical Review B 96 (Sep, 2017) 115124, 1706.00413 . 
[19] A. De Luca, M. Collura and J. De Nardis, Nonequilibrium spin transport in integrable spin chains: Persistent currents and emergence of magnetic domains, Physical Review B 96 (Jul, 2017) 020403, 1612.07265 .

[20] V. B. Bulchandani, R. Vasseur, C. Karrasch and J. E. Moore, Solvable Hydrodynamics of Quantum Integrable Systems, Phys. Rev. Lett. 119 (Dec, 2017) 220604, 1704.03466.

[21] J.-S. Caux, B. Doyon, J. Dubail, R. Konik and T. Yoshimura, Hydrodynamics of the interacting Bose gas in the Quantum Newton Cradle setup, arXiv e-prints (Nov, 2017) arXiv:1711.00873, 1711.00873 .

[22] J. De Nardis and M. Panfil, Edge Singularities and Quasilong-Range Order in Nonequilibrium Steady States, Phys. Rev. Lett. 120 (May, 2018) 217206, 1801.08079.

[23] V. B. Bulchandani, R. Vasseur, C. Karrasch and J. E. Moore, Bethe-Boltzmann hydrodynamics and spin transport in the XXZ chain, Physical Review B 97 (Jan, 2018) 045407, 1702.06146.

[24] M. Panfil and J. Pawełczyk, Linearized regime of the generalized hydrodynamics with diffusion, arXiv e-prints (May, 2019) arXiv:1905.06257, 1905.06257].

[25] A. Bastianello and A. De Luca, Integrability-protected adiabatic reversibility in quantum spin chains, Phys. Rev. Lett. 122 (Jun, 2019) 240606.

[26] A. Bastianello, V. Alba and J. Sébastien Caux, Generalized hydrodynamics with space-time inhomogeneous interactions, arXiv e-prints (Jun, 2019) arXiv:1906.01654, 1906.01654.

[27] M. Schemmer, I. Bouchoule, B. Doyon and J. Dubail, Generalized Hydrodynamics on an Atom Chip, Phys. Rev. Lett. 122 (Mar, 2019) 090601, 1810.07170.

[28] J. De Nardis, D. Bernard and B. Doyon, Hydrodynamic Diffusion in Integrable Systems, Phys. Rev. Lett. 121 (Oct, 2018) 160603, 1807.02414.

[29] E. Ilievski and J. De Nardis, Ballistic transport in the one-dimensional Hubbard model: The hydrodynamic approach, Phys. Rev. B96 (2017) 081118, 1706.05931.

[30] J. D. Nardis, D. Bernard and B. Doyon, Diffusion in generalized hydrodynamics and quasiparticle scattering, SciPost Phys. 6 (2019) 49.

[31] J. De Nardis and M. Panfil, Particle-hole pairs and density-density correlations in the Lieb-Liniger model, Journal of Statistical Mechanics: Theory and Experiment 3 (2018) 033102, 1712.06581]. 
[32] S. Gopalakrishnan, D. A. Huse, V. Khemani and R. Vasseur, Hydrodynamics of operator spreading and quasiparticle diffusion in interacting integrable systems, Phys. Rev. B98 (2018) 220303, 1809.02126.

[33] A. LeClair and G. Mussardo, Finite temperature correlation functions in integrable QFT, Nuclear Physics B 552 (1999) 624-642.

[34] B. Bertini, L. Piroli and P. Calabrese, Quantum quenches in the sinh-Gordon model: steady state and one point correlation functions, J. Stat. Mech. 1606 (2016) 063102, 1602.08269.

[35] A. Leclair and G. Mussardo, Finite temperature correlation functions in integrable QFT, Nucl. Phys. B552 (1999) 624-642, hep-th/9902075.

[36] B. Pozsgay and I. Szécsényi, LeClair-Mussardo series for two-point functions in Integrable QFT, Journal of High Energy Physics 2018 (2018) 170.

[37] B. Doyon, Finite-temperature form-factors in the free Majorana theory, J. Stat. Mech. 0511 (2005) P11006, hep-th/0506105.

[38] B. Pozsgay, Finite volume form factors and correlation functions at finite temperature. PhD thesis, -, Jul, 2009.

[39] Z. Bajnok, J. Balog, M. Lájer and C. Wu, Field theoretical derivation of Lüscher's formula and calculation of finite volume form factors, Journal of High Energy Physics 7 (2018) 174 , 1802.04021.

[40] Z. Bajnok, M. Lájer, B. Szépfalvi and I. Vona, Leading exponential finite size corrections for non-diagonal form factors, Journal of High Energy Physics 2019 (2019) 173, 1904.00492.

[41] B. Pozsgay and G. Takacs, Form factors in finite volume. II. Disconnected terms and finite temperature correlators, Nucl. Phys. B788 (2008) 209-251, 0706.3605.

[42] B. Pozsgay, Mean values of local operators in highly excited Bethe states, J. Stat. Mech. 1101 (2011) P01011, 1009.4662.

[43] Form factor approach to diagonal finite volume matrix elements in Integrable QFT, JHEP 07 (2013) 157, 1305.3373. 